\title{
Concise Commentary: The Benefits of Recycling-How Autophagy Regulation Affects Acute Pancreatitis
}

\author{
Yujiao Deng ${ }^{1} \cdot$ Zhijun Dai $^{1}$
}

Published online: 16 May 2018

๑) Springer Science+Business Media, LLC, part of Springer Nature 2018

\section{(1)

Acute pancreatitis (AP) is most commonly due to alcohol use, obstructing gallstones, or drugs. Severe acute pancreatitis (SAP) describes a subset of AP complicated by highly morbid and fatal conditions that affect extrapancreatic organs such as the systemic inflammatory response syndrome (SIRS) and multiple organ failure (MOF) [1]. Recent studies have linked the etiology of AP to dysregulated autophagy, an important physiological process in which aged cellular structures and long-lived proteins are recycled and renewed in order to maintain cellular microecological balance and cell viability [2]. Abnormal autophagy has been implicated not only in pancreatic dysfunction and pancreatic inflammatory responses, but also in pancreatic adenocarcinoma.

Genetic analysis has yielded more than 30 autophagy genes [3]: Light chain 3 (LC3), which describes microtubule-related proteins located in autophagic membranes; Beclin1, which is associated with autophagy initiation; lysosome membrane protein 2 (Lamp2), which is related to the function of autophagic lysosomes; and P62, which helps identify the degradation of the substrate. All of these proteins are considered to be markers of autophagy [4]. Impaired autophagy can promote the accumulation of pancreatic gland vacuoles, which can prematurely activate pancreatic enzymes leading to the development of AP.

In this issue of Digestive Diseases and Sciences, Wan et al. [5] explored the therapeutic effects of altering autophagy after the onset of AP in two established mouse models of AP, the arginine and caerulein + lipopolysaccharide (LPS) models, both complicated by extrapancreatic organ injury. Injections of 3-methyladenine (3-MA) and rapamycin (RAPA) were used to modulate autophagy.

In AP initiation, the activation of trypsin in pancreatic acinar cells is believed to be associated with impaired

Zhijun Dai

dzj0911@126.com

1 Department of Oncology, Second Affiliated Hospital of Xi' an Jiaotong University, Xi' an 710004, Shaanxi, China autophagy. Tong et al. [6] reported that heat shock factor (HSF)-1 attenuated the release of inflammatory cytokines induced by LPS by regulating autophagic activity. In 2010, Gukovsky et al. [7] reported that an abnormal autophagic flow exists in AP, characterized by vacuolation of the acinar cells and accumulation of trypsin in the glands, suggesting that the autophagy is related to AP. Moreover, to a certain extent, impaired autophagy can reduce the activation of abnormal trypsinogen and alleviate the severity of AP [8].

Dysregulated autophagy not only leads the development of AP, but may also contribute to the pathogenesis of pancreatic cancer. For example, Iovanna et al.[9] reported on the basis of data obtained from genetically engineered mouse model in which vacuole membrane protein (VMP)1, a protein implicated in autophagy regulation, and the oncogene Kirsten rat sarcoma viral oncogene homolog (Kras)G12D are simultaneously activated, that pancreatic autophagy promotes the development of precancerous lesions when induced by the mutated Kras homolog.

The authors concluded that impaired autophagy could increase the accumulation of reactive oxygen species (ROS) and activate NF- $\mathrm{KB}$ pathways to aggravate the disease process. 3-MA and RAPA affected serum amylase and lipase levels, and 3-MA inhibited autophagy and inflammation and downregulated NF- $\mathrm{kB}$ signaling.

\section{Compliance with ethical standards}

Conflict of interest The authors declare that they have no conflict of interest.

\section{References}

1. Singh P, Garg PK. Pathophysiological mechanisms in acute pancreatitis: current understanding. Indian J Gastroenterol. 2016;35:153-166.

2. Gukovskaya AS, Gukovsky I. Autophagy and pancreatitis. Am J Physiol Gastrointest Liver Physiol. 2012;303:G993-G1003. 
3. Farhan H, Kundu M, Ferro-Novick S. The link between autophagy and secretion: a story of multitasking proteins. Mol Biol Cell. 2017;28:1161-1164.

4. Sun A, et al. The E3 ubiquitin ligase NEDD4 is an LC3-interactive protein and regulates autophagy. Autophagy. 2017;13:522-537.

5. Wan J, et al. Regulation of autophagy affects the prognosis of mice with severe acute pancreatitis. Dig Dis Sci. (Epub ahead of print). https://doi.org/10.1007/s10620-018-5053-0.

6. Tong $\mathrm{Z}$, et al. HSF-1 is involved in attenuating the release of inflammatory cytokines induced by LPS through regulating autophagy. Shock. 2014;41:449-453.
7. Gukovsky I, Gukovskaya AS. Impaired autophagy underlies key pathological responses of acute pancreatitis. Autophagy. 2010;6:428-429.

8. Wang X, et al. Acanthopanax versus 3-methyladenine ameliorates sodium taurocholate-induced severe acute pancreatitis by inhibiting the autophagic pathway in rats. Mediators Inflamm. 2016;2016:8369704.

9. Iovanna JL. Autophagy induced during pancreatitis promotes KRAS-dependent transformation in the pancreas. Front Oncol. 2016;6:226 